\title{
AHP APPLIED TO BINARY AND TERNARY COMPARISONS
}

\author{
Iwaro Takahashi \\ University of Tsukuba
}

(Received August 19, 1988; Revised May 14, 1990)

\begin{abstract}
In this paper we confine the values of the components of comparison matrix of AHP, to two $\left(\theta, \theta^{-1}\right)$ or three $\left(\theta, 1, \theta^{-1}\right)$ distinct values. The former is called "binary case" and the latter "ternary case". Then we have some beautiful theoretical results if comparison matrices have some regularity conditions. In sports games "win, defeated, tied" just meants to ternary case, and our method serves to estimate true strength of teams from the results of "win, defeated, tied" in a leaugue tournament game.
\end{abstract}

\section{Introduction}

The intrinsic feature of AHP (Analytic Hierarchy Process) is to evaluate objects by a eigen vector of a matrix whose components are ratios taken by paired comparisons [1] [2] [3]. But often we have only binary information such as "good or bad", or "victory or defeat" in sport games or matches, by paired comparisons.

Let us call such a problem as "binary comparisons". AHP is a very useful tool to evaluate objects in binary comparisons, and in this special case we have some beasutifull results in eigen value problems $(\S 3)$. In order to deduce these results in $\S 3$, we need some basic properties in the AHP theory. These properties might be rather well known, but we do bring them here to make sure.

Further in case of sport games "tie" often occurs, and also in general evaluation we have often situations "object $i$ is as good as object $j$ " in addition to binary informations. Let us call such a problem as "ternary comparison". Say, in sport games, it is often difficult to compair a team to another, each belonging to a different league. But our ternary comparison method gives an appropriate criterion for this problem. $(\S 4)$

\section{Basic Property}

Let $E=\{1,2, \cdots, n\}$ be the set of objects $i=1,2, \cdots, n$. Let $a_{i j}(>o)$ be the ratio of evaluation of object $i$ to object $j$. Thus we have a matrix

$$
A=\left[\begin{array}{cccc}
1 & a_{12} & \cdots & a_{1 n} \\
a_{21} & 1 & \cdots & a_{2 n} \\
\vdots & \vdots & & \vdots \\
a_{n 1} & a_{n 2} & \cdots & 1
\end{array}\right]
$$

which is called comparison matrix.

We have the following well known theorem in linear algebra, 
Theorem 1. (Perron \& Frobenius)

The maximal eigen value of a matrix, whose components are all positive, is a simple positive root, and the components of the corresponding eigen vector can be all positive. [1] [4].

Thus a comparison matrix $A$ has a maximal eigen value $\lambda(>0)$ and the corresponding eigen vector

$$
\mathbf{w}=\left[w_{1} w_{2} \cdots w_{n}\right], w_{i}>o(i=1, \cdots, n)
$$

The basic idea of AHP is to take $w_{i}$ as evaluation of object $i(i=1, \cdots, n),[2],[3]$.

If we have

$$
a_{i j} a_{j i}=1(i \neq j)
$$

then $\{i, j\}$ is called consistent. If all $\{i, j\}(i, j=1, \cdots, n ; i \neq j)$ are consistent. $A$ is a reciprocal matrix and the maximal eigenvalue $\lambda$ always satisfies

$$
\lambda \geq n
$$

[1]. Hereafter we assume that all $\{i, j\}$ are consistent. Further if we have

$$
a_{i j} a_{j k} a_{k i}=1(i \neq j \neq k \neq i)
$$

then $\{i, j, k\}$ is called consistent. Then we have the following theorem.

Theorem 2. The necessary and sufficient condition for $a_{i j}$ (components of a comparison matrix $A$ ) to be represented as

$$
a_{i j}=w_{i} / w_{j}(i, j=1, \cdots, n)
$$

by some positive value $w_{i}(i=1, \cdots, n)$ is that all $\{i, j, k\}(i, j, k=1, \cdots, n ; i \neq j \neq k \neq i)$ are consistent.

Proof: It is clear that if (2.5) is valid then we have (2.4) for all $\{i, j, k\}$. So we have only to show the validity of $(2.5)$ from $(2.4)$. Let us take $w_{1}, \cdots, w_{n}$ as

$$
w_{1}=1, w_{2}=a_{21}, w_{3}=a_{31}, \cdots, w_{n}=a_{n 1}
$$

then for any $i, j$ we have

$$
a_{i j} a_{j 1} a_{1 i}=1, a_{1 i}=1 / a_{i 1}
$$

from(2.4) and (2.2). So we have

$$
a_{i j}=\frac{1}{a_{j 1} \cdot a_{1 i}}=\frac{1}{w_{j} \cdot 1 / w_{i}}=\frac{w_{i}}{w_{j}}
$$

If components $a_{i j}$ of comparison matrix $A$ satisfy (2.5), then $E=\{1,2, \cdots, n\}$ (or $A$ it self) is called consistent. Thus theorem 2 states that the necessary and sufficient condition of consistency of $E=\{1,2, \cdots, n\}$ is that any 3 -set $\{i, j, k\} \subseteq E$ is consistent.

The following theorem is fundamental and well known [1] [3], but we will give our own elementary proof for it.

Theorem 3. If an nxn comparison matrix $A=\left[a_{i j}\right]$ is consistent then the maximal eigenvalue of $A$ is equal to $n$, and its eigenvector is

$$
\mathbf{w}=\left[1, a_{21}, \cdots, a_{n 1}\right]
$$


Proof: The proper equation of $A$ is

$$
\left|\begin{array}{cccc}
1-\lambda & w_{1} / w_{2} & \cdots & w_{1} / w_{n} \\
w_{2} / w_{1} & 1-\lambda & \cdots & w_{2} / w_{n} \\
\vdots & \vdots & & \vdots \\
w_{n} / w_{1} & w_{n} / w_{2} & \cdots & 1-\lambda
\end{array}\right|=0
$$

Multipling $i$-th column by $w_{i}$ and dividing $j$-th column by $w_{j}$, we have

$$
\left|\begin{array}{cccc}
1-\lambda & 1 & \cdots & 1 \\
1 & 1-\lambda & \cdots & 1 \\
\vdots & \vdots & & \vdots \\
1 & 1 & \cdots & 1-\lambda
\end{array}\right|=0
$$

Adding $i$-th column $(i=2,3, \cdots, n)$ to the first column we have

$$
\left|\begin{array}{cccc}
n-\lambda & 1 & \cdots & 1 \\
n-\lambda & 1-\lambda & \cdots & 1 \\
\vdots & \vdots & & \vdots \\
n-\lambda & 1 & \cdots & 1-\lambda
\end{array}\right|=(n-\lambda)\left|\begin{array}{cccc}
1 & 1 & \cdots & 1 \\
1 & 1-\lambda & \cdots & 1 \\
\vdots & \vdots & & \vdots \\
1 & 1 & \cdots & 1-\lambda
\end{array}\right|=0
$$

Subtracting the first row from all other rows we have

$$
(n-\lambda)\left|\begin{array}{cccc}
1 & 1 & \cdots & 1 \\
0 & -\lambda & \cdots & 0 \\
\vdots & \vdots & & \vdots \\
0 & 0 & \cdots & -\lambda
\end{array}\right|= \pm(n-\lambda) \lambda^{n-1}=0
$$

which states that eigenvalues of $A$ are $n$ and zero, so the maximal eigenvalue is $n$.

Further multipling $\mathbf{w}$ (represented by (2.7)) by $A$ we have $A \mathbf{w}=n \mathbf{w}$. So $\mathbf{w}$ is the eigen vector for $\lambda=n$.

\section{Binary Comparisons}

We consider, say, sport games or matches among $n$ teams $\{1,2, \cdots, n\}$. In such case the information taken from a match between team $i$ and $j$ is only "victory of defeat". If team $i$ wins and $j$ loses then let $a_{i j}$ and $a_{j i}$ be

$$
a_{i j}=\theta, a_{j i}=1 / \theta
$$

with a parameter $\theta(>1)$. Then from a league tournament in $\{1,2, \cdots, n\}$ we have a comparison matrix $A=\left[a_{i j}\right]$, whose non diagonal elements are $\theta$ or $1 / \theta$. Let us call such a problem a "binary comparison (with parameter $\theta$ )".

In a general evaluation problem if we require only "good or bad" on paired comparison this becomes also a binary comparison.

In a binary comparison on $E=\{1,2, \cdots, n\}$ if the condition

$$
a_{i j}>1, a_{j k}>1 \text { implies } a_{i k}>1(i \neq j \neq k \neq i)
$$


holds then let $\{i, j, k\}(\subseteq E)$ be called "logically consistent". Note that if $\{i, j, k\}$ is consistent then this is logically consistent, but the inverse is not necessarily true. If any 3 -set $\{i, j, k\} \subseteq$ $E$ is logically consistent then let "the binary comparison on $E$ be called logically consistent."

We construct a directed graph corresponding to a comparison matrix $A=\left[a_{i j}\right]$ of a binary comparison on $E=[\{1,2, \cdots, n\}$ by the following way; we have a directed arc connecting point $i$ to $j$ iff $a_{i j}>1$ (that is $a_{i j}=\theta$ ), being $E$ the set of points of the graph. (see $E x 1, E x 2 \cdots)$.

Then the graph of a logically consistent binary comparison of $E$ is acyclic and any two points have a directed arc, so $E$ is a totally ordered set, where $i$ precedes $j$ iff $a_{i j}>1$. Let us renumber points in $E$ along this order, then the comparison matrix $A$ is written as

$$
A=\left[\begin{array}{ccccc}
1 & \theta & \theta & \cdots & \theta \\
1 / \theta & 1 & \theta & \cdots & \theta \\
1 / \theta & 1 / \theta & 1 & \cdots & \theta \\
\vdots & \vdots & \vdots & & \vdots \\
1 / \theta & 1 / \theta & 1 / \theta & \cdots & 1
\end{array}\right]
$$

Theorem 4. A comparison matrix $A$ (represented in the from (3.3)) of a logically consistent binary comparison has the maximal eigen value

$$
\lambda=1+\theta\left(w+w^{2}+\cdots+w^{n-1}\right)
$$

and the corresponding eigen vector

$$
\mathbf{w}=\left[1, w, w^{2}, \cdots, w^{n-1}\right]
$$

where

$$
\theta^{2} w^{n}=1
$$

Proof: Firstly we show that

$$
A \mathbf{w}=\lambda \mathbf{w}
$$

that is, $\mathbf{w}$ is the eigen vector corresponding to $\lambda$. Let $(A \mathbf{w})_{i}$ be the $i$-th component of $A \mathbf{w}$, then we have

$$
\begin{gathered}
(A \mathbf{w})_{1}=1+\theta\left(w+w^{2}+w^{n-1}\right)=\lambda \\
(A \mathbf{w})_{i}=\left(1+w+\cdots+w^{i-2}\right) / \theta+w^{i-1}+\theta\left(w^{i}+\cdots+w^{n-1}\right) \\
(i=2, \cdots, n, \text { the third term vanishes when } i=n)
\end{gathered}
$$

On the other hand we have

$$
\begin{aligned}
\lambda w^{i-1} & =w^{i-1}+\theta\left(w^{i}+\cdots+w^{n-1}+w^{n}+\cdots+w^{n+i-2}\right) \\
& =w^{i-1}+\theta\left(w^{i}+\cdots+w^{n-1}\right)+\theta w^{n}\left(1+\cdots+w^{i-2}\right) \\
& =w^{i-1}+\theta\left(w^{i}+\cdots+w^{n-1}\right)+1 / \theta\left(1+\cdots+w^{i-2}\right),
\end{aligned}
$$

which is equal to (3.8), so we have (3.7).

Next we show that $\lambda$ is the maximal eigen value of $A$. Let the maximal eigen value of $A$ be $\rho$, then the transposed matrix $A^{\prime}$ of $A$ has the same maximal eigen value $\rho$. From Theorem $1, \rho$ is positive and the corresponding eigen vector $\mathbf{x}$ of $A^{\prime}$ is positive (that is, its components are all positive). 
Now we have

$$
\rho\left(\mathbf{x}^{\prime} \mathbf{w}\right)=(\rho x)^{\prime} \mathbf{w}=\left(A^{\prime} \mathbf{x}\right)^{\prime} \mathbf{w}=\mathbf{x}^{\prime} A \mathbf{w}=\lambda\left(\mathbf{x}^{\prime} \mathbf{w}\right)
$$

and both $\mathbf{x}$ and $\mathbf{w}$ are positive vectors so $\mathbf{x}^{\prime} \mathbf{w}$ cannot vanish, therefore we have $\lambda=\rho$.

Example 1 For a comparison matrix and its graph shown below

$$
\mathrm{A}=\left[\begin{array}{lll}
1 & \theta & \theta \\
1 / \theta & 1 & \theta \\
1 / \theta & 1 / \theta & 1
\end{array}\right]
$$

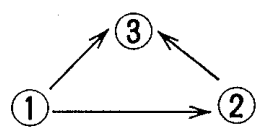

we have

$\lambda=3.0536 \mathbf{w}=[1,0.63,0.3968](\theta=2)$, from Theorem 4 . Instead $\mathbf{w}$ we often use standardized vector $\overline{\mathbf{w}}$, the sum of whose elements is equal to unity. Thus we have

$$
\overline{\mathbf{w}}=[0.4934,0.3108,0.1958]
$$

Of course we often encounter non-consistent binary comparisons in the real world. Thought $A$ is not consistent the evaluation based on the eigen vector gives an appropriate criterion.

\section{Example 2.}

$$
\begin{gathered}
A=\left[\begin{array}{ccc}
1 & \theta & 1 / \theta \\
1 / \theta & 1 & \theta \\
\theta & 1 / \theta & 1
\end{array}\right] \\
\lambda=3.5 \mathbf{w}=[1,1,1](\theta=2)
\end{gathered}
$$

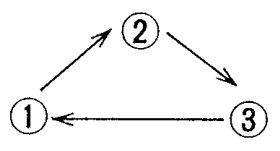

\section{Example 3.}

$$
\begin{aligned}
A= & \begin{array}{l}
1 \\
2 \\
3
\end{array}\left[\begin{array}{llll}
1 & 2 & 3 & 4 \\
1 / \theta & 1 & 1 / \theta & \theta \\
\theta & 1 / \theta & 1 & 1 / \theta \\
1 / \theta & 1 / \theta & \theta & 1
\end{array}\right] \\
\lambda & =4.644(\theta=2) \\
\mathbf{w} & =[1,0.9396,0.7724,0.6899] \\
& \quad \mathbf{w}=[0.2940,0.2762,0.2133,0.2028] .
\end{aligned}
$$

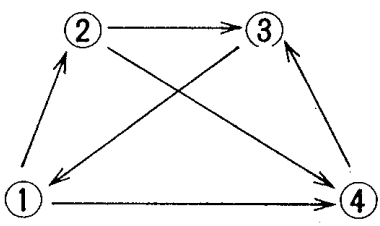

Let $A$ represent scores of a league tournament on $E=\{1,2,3,4\}$. Both teams 1 and 2 win two matches and lose one, but team 1 gets higher evaluation then team 2 . The reason is rather complicated but mainly is that team 1 defeats team 2 . 


\section{Ternary Comparisons}

In case of sport games "tie" often occurs. We assume that if team $i$ ties team $j$ then $a_{i j}=1$, then nondiagonal elements of the comparison matrix $A=\left[a_{i j}\right]$ are $\theta, 1 / \theta$ or 1 . Let such a problem be called a "ternary comparison". In general evaluation problem if object $i$ is as good as object $j$, or $i$ is equivalent to $j$ then $a_{i j}$ is to be unity, which leads to a ternary comparison. In the graph representation of a ternary comparison, if $a_{i j}=1$ then point $i$ is to be connected to point $j$ with an undirected arc.

In this section we will give an evaluation criterion $n \overline{\mathbf{w}}$ and show how this is appropriate for evaluation among different groups of objects through various examples.

\section{Example 4.}

$A=\left[\begin{array}{lll}1 & 1 & \theta \\ 1 & 1 & 1 \\ 1 / \theta & 1 & 1\end{array}\right]$

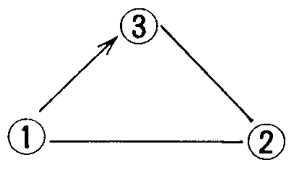

$$
\begin{aligned}
& \lambda=3.054(\theta=2) \\
& \mathbf{w}=[1,0.7937,0.63] \\
& \overline{\mathbf{w}}=[0.4126,0.3275,0.26]
\end{aligned}
$$

(Incidentally we have $\lambda=1+w+\theta w^{2}, \theta w^{3}=1$,

$$
\left.\mathbf{w}=\left[1, w, w^{2}\right]\right)
$$

\section{Example 5.}

$$
\begin{aligned}
& A=\left[\begin{array}{lll}
1 & \theta & 1 \\
1 / \theta & 1 & \theta \\
1 & 1 / \theta & 1
\end{array}\right] \\
& \lambda=3.217(\theta=2) \\
& \mathbf{w}=[1,0.7937,0.63] \\
& \left(\lambda=1+\theta w+w^{2}, \theta w^{3}=1, \mathbf{w}=\left[1, w, w^{2}\right]\right)
\end{aligned}
$$

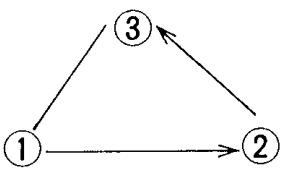

Example 6. (consistent)

$$
\begin{aligned}
A=\left[\begin{array}{lll}
1 & 1 & \theta \\
1 & 1 & \theta \\
1 / \theta & 1 / \theta & \theta
\end{array}\right] \\
\qquad \begin{array}{r}
\lambda=3, \mathbf{w}=[1,1,1 / \theta] \\
\overline{\mathbf{w}}=\left[\begin{array}{lll}
0.4 & 0.4 & 0.2
\end{array}\right](\theta=2)
\end{array}
\end{aligned}
$$

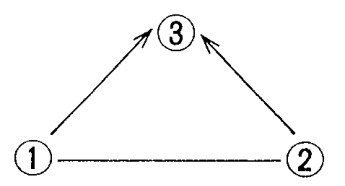




\section{Example 7.}

$$
A=\frac{1}{2}+\left[\begin{array}{llll}
1 & 2 & 3 & 4 \\
1 & 1 & \theta & 1 \\
1 / \theta & 1 & 1 & \theta \\
1 & 1 / \theta & 1 & \theta \\
1 / \theta & 1
\end{array}\right]
$$

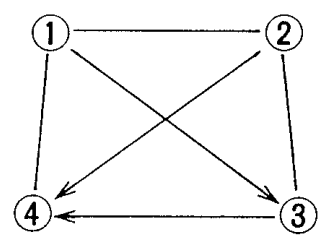

$$
\begin{aligned}
& \lambda=4.186(\theta=2) \\
& \mathbf{w}=[1,0.9443,0.8248,0.5916] \\
& \overline{\mathbf{w}}=[0.2976,0.2810,0.2454,0.1760] .
\end{aligned}
$$

Both teams 1 and 2 win one match and tie two matches, but team 1 gets higher evaluation.

\section{Example 8}

$A=\begin{aligned} & 1 \\ & 2 \\ & 3 \\ & 4\end{aligned}\left[\begin{array}{llll}1 & 2 & 3 & 4 \\ 1 & 1 & 1 & \theta \\ 1 / \theta & 1 & 1 & \theta \\ 1 / \theta & 1 & 1 / \theta & 1\end{array}\right]$

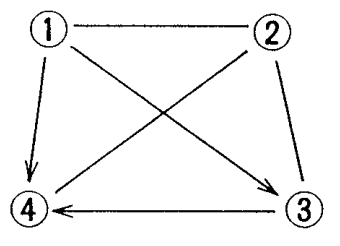

$$
\begin{aligned}
& \lambda=4.121,(\theta=2) \\
& \mathbf{w}=[1,0.7071,0.7071,0.5] \\
& \overline{\mathbf{w}}=[0.3431,0.2426,0.2426,0.1716]
\end{aligned}
$$

Team 2 ties all matches and team 3 wins one and loses one, but they have the same evaluation. (Let $\theta=4$ then we have $\lambda=4.5, \mathbf{w}=[1,0.5,0.5,0.25]$ )

\section{Example 9}

$$
\begin{aligned}
& A=\begin{array}{l}
1 \\
2 \\
3 \\
4
\end{array}\left[\begin{array}{llll}
1 & \theta & 1 & 1 \\
1 / \theta & 1 & \theta & 1 \\
1 & 1 / \theta & 1 & \theta \\
1 & 1 & 1 / \theta & 1
\end{array}\right] \\
& \lambda=4.3101(\theta=2) \\
& \mathbf{w}=[1,0.8787,0.8559,0.6988] \\
& \overline{\mathbf{w}}=[0.2913,0.2559,0.2493,0.2035]
\end{aligned}
$$

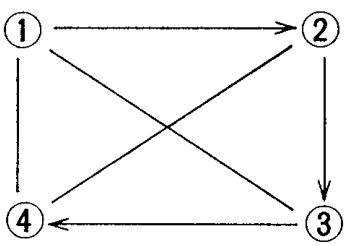

Generally each component of $n \overline{\mathbf{w}}$ is appropriate criterion on the evaluation among different league tournaments. For example, we have the values of $n \overline{\mathbf{w}}$ for Ex. 4, Ex. 6, Ex. 7 and Ex. 8, 
Ex. $4: 3 \overline{\mathbf{w}}=[1.238,0.9825,0.78]$

Ex. $6: 2 \overline{\mathbf{w}}=[1.2,1.2,0.6]$

Ex. $7: 4 \overline{\mathbf{w}}=[1.1902,1.124,0.9817,0.7041]$

Ex. $8: 4 \overline{\mathbf{W}}=[1.375,0.972,0.972,0.688]$

In Ex. 6 team 1 wins one, ties one and gets evaluation of 1.2. while in Ex. 7 team 1 wins one, ties two and gets evaluation of 1.1902 slightly smaller than the former. Further team 3 in Ex. 7 and team 3 in Ex. 8 win one, lose one and tie one, but the former gets 0.9817 and the latter 0.972 slightly smaller than the latter.

It can be said that these evaluation are very close to our intuitive evaluation for abilities of these teams.

\section{References}

[1] Saaty, T.L.: A Scaling Method for Priorities in Hierarchical Structures. J. of Mathematical Psychology, Vol. 15, (1977), 234-281.

[2] Saaty, T.L.: The Analytic Hierarchy Process. Mc Graw-Hill 1980

[3] Manabe, R.: AHP (Analytic Hierarchy Process) (in Japanese), Communications of the O.R. Society of Japan, Vol. 31, (1986), 475-478.

[4] Ito, N \& others: Matrices and their Applicalins (in Japanese), Kinokuniya Publishing Co. 1987

Iwaro Takahashi

Socio-Economic Planning, University of Tsukuba

Tsukuba-City, Ibaraki 305, Japan 